\title{
THE ADJOINTS OF DIFFERENTIABLE MAPPINGS
}

\author{
S. YAMAMURO \\ (Received 14 December 1966)
}

The notion of symmetric (non-linear) mappings has been introduced by Vainberg [3, p. 56]. However, symmetric mappings of this type have not played any important rôle in non-linear functional analysis. Naturally, as in the case of linear mappings, the symmetric mappings should be defined in such a way that they are easy to handle and belong to the most elementary class of non-linear mappings.

In this paper, we shall introduce the notion of adjoint mappings of non-linear mappings and define symmetric mappings as the mappings which coincide with their adjoints. It will be seen that a mapping is symmetric if and only if it is potential. (See Theorem 1.) This means that our definition gives a natural generalization of the notion of symmetry for linear mappings, because it is evident that a linear mapping is symmetric if and only if it is potential.

An extensive study on the potential mappings can be found in Vainberg's book [3]. We shall spend most of this paper for the study on the notion of adjoint mappings.

\section{Preliminaries}

Let $\boldsymbol{E}$ be a real Hilbert space. A mapping $f$ of $\boldsymbol{E}$ into itself is said to be (Fréchet-)differentiable at $a \in \boldsymbol{E}$ if there exists a continuous linear mapping $l$ of $\boldsymbol{E}$ into $\boldsymbol{E}$ such that

where

$$
f(a+x)-f(a)=l(x)+r(a, x) \quad \text { for every } x \in E
$$

$$
\lim _{\|x\| \rightarrow 0}\|r(a, x)\| /\|x\|=0 .
$$

The linear mapping $l$ is determined uniquely and depends on the element $a$. We call it the derivative of $f$ at $a$ and denote it by $f^{\prime}(a)$.

If a mapping $f$ is differentiable at every point of $\boldsymbol{E}, f$ is said to be differentiable. In this case, $f^{\prime}(x)$ is a mapping of $\boldsymbol{E}$ into the set $\mathscr{L}$ of all continuous linear mappings of $\boldsymbol{E}$ into $\boldsymbol{E}$. As is well known, the set $\mathscr{L}$ is a Banach algebra with the norm:

$$
\|l\|=\sup _{\substack{\|x\|=1 \\ \mathbf{3 9 7}}}\|l(x)\| \quad \text { for every } l \in \mathscr{L} .
$$


If the mapping $f^{\prime}(x)$ is continuous with respect to this norm topology, $f$ is said to be continuously differentiable.

Throughout this paper, we denote by $\mathscr{D}$ the set of all continuously differentiable mappings $f$ such that $f(0)=0$. Real numbers are denoted by the Greek letters.

\section{2. *-admissibility}

A mapping $f \in \mathscr{D}$ is said to be ${ }^{*}$-admissible if there exists $g \in \mathscr{D}$ such that

$$
g^{\prime}(x)=\left(f^{\prime}(x)\right)^{*} \quad \text { for every } x \in E,
$$

where $\left(f^{\prime}(x)\right)^{*}$ denotes the adjoint of the linear mapping $f^{\prime}(x)$. In other words, $f \in \mathscr{D}$ is *-admissible if there exists $g \in \mathscr{D}$ such that

$$
\left(g^{\prime}(x)(y), z\right)=\left(y, f^{\prime}(x)(z)\right)
$$

for any $x, y$ and $z$ in $\boldsymbol{E}$. $\left(f^{\prime}(x)(z)\right.$ is the value of $f^{\prime}(x)$ at $z$, and $\left(y, f^{\prime}(x)(z)\right)$ is the inner product of $y$ and $f^{\prime}(x)(z)$.)

(2) If $f \in \mathscr{D}$ is *-admissible, the mapping $g$ in (1) is determined uniquely.

To prove this, we have only to prove generally that, if $f, g \in \mathscr{D}$ and

$$
f^{\prime}(x)=g^{\prime}(x) \quad \text { for every } x \in E,
$$

we have $f(x)=g(x)$ for every $x \in E$. Let us consider abstract functions $f(\xi x)$ and $g(\xi x)$ of real variable $\xi$. Since

$$
\frac{d}{d \xi} f(\xi x)=f^{\prime}(\xi x)(x) \text { and } \frac{d}{d \xi} g(\xi x)=g^{\prime}(\xi x)(x),
$$

it follows from [Theorem 2.7, p. 34, [3]] that

$$
f(x)=\int_{0}^{1} f^{\prime}(\xi x)(x) d \xi=\int_{0}^{1} g^{\prime}(\xi x)(x) d \xi=g(x) .
$$

In the sequel, we denote this uniquely determined mapping by $f^{*}$ and call it the adjoint of $f$.

A linear mapping $l \in \mathscr{L}$ is always *-admissible because $\mathscr{L} \subset \mathscr{D}$ and

$$
l^{\prime}(x)=l \quad \text { for every } x \in \boldsymbol{E},
$$

and $l^{*}$ defined in this way coincides with the usual adjoint.

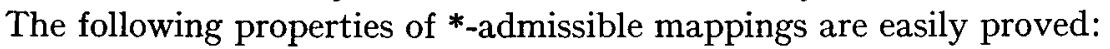

(3) If $f$ is *-admissible, $f^{*}$ is also *-admissible and

$$
\left(f^{*}\right)^{*}=f \text {. }
$$




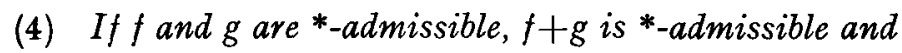

$$
(\alpha f+\beta g)^{*}=\alpha f^{*}+\beta g^{*} .
$$

(5) If $f$ is *-admissible,

$$
f^{*}(x)=\int_{0}^{1}\left(f^{\prime}(\xi x)\right)^{*}(x) d \xi \quad \text { for every } x \in \boldsymbol{E} .
$$

The following property will be used frequently.

(6) If $f$ is *-admissible,

$$
(f(x), x)=\left(f^{*}(x), x\right) \quad \text { for every } x \in \boldsymbol{E} .
$$

In fact, by making use of properties of the abstract integral $(\S 2$, Chapter I, [3]), we have

$$
\begin{aligned}
(f(x), x) & =\left(\int_{0}^{1} f^{\prime}(\xi x)(x) d \xi, x\right) \\
& =\int_{0}^{1}\left(f^{\prime}(\xi x)(x), x\right) d \xi \\
& =\int_{0}^{1}\left(\left(f^{\prime}(\xi x)\right)^{*}(x), x\right) d \xi \\
& =\left(\int_{0}^{1}\left(f^{\prime}(\xi x)\right)^{*}(x) d \xi, x\right)=\left(f^{*}(x), x\right) .
\end{aligned}
$$

\section{Symmetry and skew-symmetry}

A mapping $f \in \mathscr{D}$ is said to be symmetric if it is *admissible and $f=f^{*}$. If $f$ is *-admissible and $f^{*}=-f$, it is said to be skew-symmetric.

From (3) and (4) it follows immediately that, if $f$ is *admissible, $f+f^{*}$ is symmetric and $f-f^{*}$ is skew-symmetric.

The following theorem is a paraphrase of [Theorem 5.1, p. 56, [3]].

THEOREM 1. A mapping $f$ is symmetric if and only if it is potential; in other words, $f$ is symmetric if and only if there exists a real-valued function $\phi(x)$ on $\boldsymbol{E}$ such that

$$
\phi(x+y)-\phi(x)=(f(x), y)+r(x, y)
$$

for any $x$ and $y$ in $E$ and

$$
\lim _{\|\boldsymbol{y}\| \rightarrow 0}|r(x, y)| /\|y\|=0 .
$$

Next, we give a characterization for skew-symmetric mappings.

THEOREM 2. A mapping $f$ is skew-symmetric if and only if it is linear and $(f(x), x)=0$ for every $x \in \boldsymbol{E}$.

Proof. Let $f \in \mathscr{D}$ be skew-symmetric. Then, it follows from (6) that

$$
(f(x), x)=\left(f^{*}(x), x\right)=-(f(x), x)
$$


for every $x \in \boldsymbol{E}$. Therefore, $(f(x), x)=0$ for every $x \in \boldsymbol{E}$. Thus, we have only to prove that $f$ is linear. At first, we prove that, if $f$ is skew-symmetric, $(f(x+\xi y), y)$ is constant with respect to $\xi$. In fact,

$$
\begin{aligned}
\frac{d}{d \xi}(f(x+\xi y), y) & =\left(f^{\prime}(x+\xi y)(y), y\right) \\
& =\left(\left(f^{\prime}(x+\xi y)\right) *(y), y\right) \\
& =\left(\left(f^{*}\right)^{\prime}(x+\xi y)(y), y\right) \\
& =-\left(f^{\prime}(x+\xi y)(y), y\right) \\
& =-\frac{d}{d \xi}(f(x+\xi y), y),
\end{aligned}
$$

hence it follows that

$$
\frac{d}{d \xi}(f(x+\xi y), y)=0
$$

for every $\xi$.

Therefore, especially, we have

Similarly,

$$
(f(x+y), y)=(f(x), y) \quad \text { for any } x \text { and } y \text { in } E .
$$

$$
(f(x+y), x)=(f(y), x) \quad \text { for any } x \text { and } y \text { in } \boldsymbol{E} .
$$

On the other hand, we have

$$
0=(f(x+y), x+y)=(f(x+y), x)+(f(x+y), y) .
$$

These three equalities imply that

$$
(f(x), y)=-(f(y), x) \quad \text { for any } x \text { and } y \text { in } \boldsymbol{E},
$$

from which the linearity of $f$ follows.

Conversely, if $l$ is linear and $(l(x), x)=0$ for any $x \in E$, we have

$$
(l(x), y)+(l(y), x)=(l(x+y), x+y)-(l(x), x)-(l(y), y)=0,
$$

from which it follows that $l^{*}=-l$.

\section{Conditions for *-admissibility}

The adjoints of non-linear mappings, unlike the adjoints of continuous linear mappings, cannot always be defined. Theorem 1 suggests the existence of close connection between *-admissibility and potentiality. The following theorem makes the connection clear.

THEOREM 3. $f \in \mathscr{D}$ is *-admissible if and only if there exists a skeresymmetric mapping $l \in \mathscr{L}$ such that $f+l$ is potential. 
Proof. If $f \in \mathscr{D}$ is *-admissible, we have that

$$
f=\frac{1}{2}\left(f+f^{*}\right)+\frac{1}{2}\left(f-f^{*}\right),
$$

$\frac{1}{2}\left(f+f^{*}\right)$ is symmetric (= potential by Theorem 1 ) and $\frac{1}{2}\left(f-f^{*}\right)$ is skewsymmetric. Therefore, we can take $\frac{1}{2}\left(f-f^{*}\right)$ as the mapping $l$ in the theorem. Conversely, let us assume that $f \in \mathscr{D}$ and $f+l$ is potential for some skewsymmetric mapping $l \in \mathscr{L}$. Then, since $f+l$ and $l$ are ${ }^{*}$-admissible, it follows from (4) that $f$ is ${ }^{*}$-admissible.

It should be remembered that, if $f$ is *-admissible, the skew-symmetric mapping in the above theorem can be determined uniquely. In fact, if there are two skew-symmetric mappings $l_{1}$ and $l_{2}$ such that $f+l_{1}$ and $f+l_{2}$ are symmetric, $l_{1}-l_{2}=\left(f+l_{1}\right)-\left(f+l_{2}\right)$ is also symmetric. Therefore,

$$
l_{1}-l_{2}=\left(l_{1}-l_{2}\right)^{*}=l_{1}^{*}-l_{2}^{*}=l_{2}-l_{1},
$$

from which it follows that $l_{1}=l_{2}$.

Thus, since non-zero skew-symmetric mappings are not symmetric, it is meant by

$$
f=\frac{1}{2}\left(f+f^{*}\right)+\frac{1}{2}\left(f-f^{*}\right)
$$

that the set of all *-admissible mappings is the direct sum of the set of all symmetric mappings and the set of all skew-symmetric mappings. In other words, if we put

$$
\begin{aligned}
f_{s} & =\frac{1}{2}\left(f+f^{*}\right) \text { and } l_{f}=\frac{1}{2}\left(f-f^{*}\right), \\
f & =t_{s}+l_{f}
\end{aligned}
$$

is the unique expression of a ${ }^{*}$-admissible mapping $f$ as the sum of a symmetric mapping and a skew-symmetric mapping, and it is easy to see that

$$
f^{*}=f-2 l_{\rho} .
$$

Now, we give another characterization for the *-admissibility.

TheOREM 4. $f \in \mathscr{D}$ is *-admissible if and only if

is independent of $x \in \boldsymbol{E}$.

$$
f^{\prime}(x)-\left(f^{\prime}(x)\right)^{*}
$$

Proof. If $f$ is *-admissible, we have by (7) that

$$
f-f^{*}=2 l_{f} \text {. }
$$

Therefore, since $l_{f}$ is a linear mapping,

$$
f^{\prime}(x)-\left(f^{\prime}(x)\right)^{*}=\left(f-f^{*}\right)^{\prime}(x)=2 l_{f}^{\prime}(x)=2 l_{f},
$$

which means that $f^{\prime}(x)-\left(f^{\prime}(x)\right)^{*}$ is independent of $x \in E$. Conversely, if $f^{\prime}(x)-\left(f^{\prime}(x)\right) *$ is independent of $x \in E$, we can put 


$$
f^{\prime}(x)-\left(f^{\prime}(x)\right)^{*}=l
$$

Then, for $g=t-l$, we have

$$
g^{\prime}(x)=(f-l)^{\prime}(x)=f^{\prime}(x)-l=\left(f^{\prime}(x)\right)^{*} .
$$

Therefore, by the definition of the *-admissibility, $f$ is $*_{\text {-admissible. }}$

The following fact follows immediately from this theorem.

(8) If $f \in \mathscr{D}$ is *-admissible and $f^{\prime}(a)$ is symmetric for some $a \in \boldsymbol{E}$, then $f$ itself is symmetric.

In fact, if $f$ satisfies this condition, we have $f=f_{s}$, because

$$
l_{f}=\frac{1}{2}\left(f^{\prime}(a)-\left(f^{\prime}(a)\right)^{*}\right)=0 .
$$

In particular, a ${ }^{*}$-admissible mapping $f$ is symmetric if $f^{\prime}(a)=0$ for some $a \in \boldsymbol{E}$.

\section{5. *-admissibility for the twice differentiable mappings}

For the twice differentiable mappings, we can have a simpler criterion for the *admissibility. Let $f$ be twice continuously differentiable; in other words, there exists a continuous linear mapping $f^{\prime \prime}(x)$ of $\boldsymbol{E}$ into $\mathscr{L}$ such that

$$
f^{\prime}(x+y)-f^{\prime}(x)=f^{\prime \prime}(x)(y)+r(x, y)
$$

for every $x$ and $y$ in $\boldsymbol{E}$, where

$$
\lim _{\|y\| \rightarrow 0}\|r(x, y)\| /\|y\|=0,
$$

and $f^{\prime \prime}(x)$ is continuous with respect to $x \in \boldsymbol{E}$. (Therefore, $f^{\prime \prime}(x)(y) \in \mathscr{L}$ for any $x$ and $y$ in $\boldsymbol{E}$.)

THEOREM 5. Let $f$ be twice continuously differentiable. Then, $f$ is

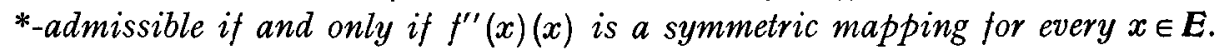

Proof. Let $f$ be ${ }^{*}$-admissible. Then, by Theorem 4,

$$
\begin{aligned}
f^{\prime \prime}(x)(x)- & \left(f^{\prime \prime}(x)(x)\right)^{*} \\
& =\lim _{\varepsilon \rightarrow 0} \frac{1}{\varepsilon}\left[\left(f^{\prime}(x+\varepsilon x)-f^{\prime}(x)\right)-\left(f^{\prime}(x+\varepsilon x)-f^{\prime}(x)\right)^{*}\right] \\
& =\lim _{\varepsilon \rightarrow 0} \frac{1}{\varepsilon}\left[\left(f^{\prime}(x+\varepsilon x)-\left(f^{\prime}(x+\varepsilon x)^{*}\right)-\left(f^{\prime}(x)-\left(f^{\prime}(x)\right)^{*}\right)\right]\right. \\
& =0 .
\end{aligned}
$$

Conversely, if $f^{\prime \prime}(x)(x)$ is symmetric for every $x \in \boldsymbol{E}$, since 
we have

$$
f^{\prime}(x)-f^{\prime}(0)=\int_{0}^{1} f^{\prime \prime}(\xi x)(x) d \xi
$$

$$
\begin{aligned}
f^{\prime}(x)-\left(f^{\prime}(x)\right)^{*}= & \int_{0}^{1} f^{\prime \prime}(\xi x)(x) d \xi+f^{\prime}(0) \\
& -\left(\int_{0}^{1} f^{\prime \prime}(\xi x)(x) d \xi+f^{\prime}(0)\right)^{*} \\
= & \int_{0}^{1} f^{\prime \prime}(\xi x)(x) d \xi+f^{\prime}(0) \\
& -\int_{0}^{1}\left(f^{\prime \prime}(\xi x)(x)\right)^{*} d \xi-\left(f^{\prime}(0)\right)^{*} .
\end{aligned}
$$

Therefore, by Theorem $4, f$ is $*$-admissible.

\section{Products of $*$-admissible mappings}

The product $f g$ of two mappings $f \in \mathscr{D}$ and $g \in \mathscr{D}$ is defined by

$$
(f g)(x)=f(g(x)) \quad \text { for every } x \in \boldsymbol{E} .
$$

It is well-known that $f g \in \mathscr{D}$ and

$$
(f g)^{\prime}(x)=f^{\prime}(g(x)) g^{\prime}(x) \quad \text { for every } x \in \boldsymbol{E} .
$$

As is easily seen from this equality, the product of two *admissible mappings is not always *-admissible. In this section, we hall take a deeper look into this fact. We begin with some lemmas.

(9) Let $l \in \mathscr{L}$ and $\boldsymbol{E}_{x}=\{\xi x \mid-\infty<\xi<\infty\}$ be a one-dimensional closed subspace generated by a single element $x \in \boldsymbol{E}$. If $l(x) \in \boldsymbol{E}_{x}$ for every $x \in \boldsymbol{E}$, there exists a number $\alpha$ such that $l=\alpha 1$, where 1 is the identity mapping.

Proof. By the assumption, there exists a real-valued function $\phi(x)$ such that

$$
l(x)=\phi(x) x \quad \text { for every } x \in \boldsymbol{E} .
$$

We have only to prove that $\phi(x)$ is a constant function. Let $x$ and $y$ are arbitrary non-zero elements. If $(x, y)=\mathbf{0}$, since

we have

$$
\begin{aligned}
\phi(x) x+\phi(y) y & =l(x)+l(y)=l(x+y) \\
& =\phi(x+y) x+\phi(x+y) y,
\end{aligned}
$$

$$
(\phi(x) x, x)+(\phi(y) y, x)=(\phi(x+y) x, x)+(\phi(x+y) y, x),
$$

hence it follows that

$$
\phi(x)=\phi(x+y)
$$

If $y \in E_{x}$, since $y=\alpha x$ for some $\alpha$ and 
we have

$$
\phi(y) y=l(y)=l(\alpha x)=\alpha \phi(x) x=\phi(x)(\alpha x)=\phi(x) y,
$$

$$
\phi(x)=\phi(y)
$$

Now generally, since $E_{x}$ is a closed linear subspace, there exist $y_{1} \in E_{x}$ and $y_{2} \in E_{x}^{\perp}=\{z \in E \mid(x, z)=0\}$ such that

Then,

$$
y=y_{1}+y_{2} \text {. }
$$

$$
\begin{aligned}
\phi(y) & =\phi\left(y_{1}+y_{2}\right) \\
& =\phi\left(y_{1}\right) \\
& =\phi(x)
\end{aligned}
$$

which means that $\phi(x)$ is a constant function.

(12) Let $f$ be symmetric. If lf is symmetric for every $l \in \mathscr{L}$, then $f=0$.

Proof. From the assumption that $(l f)^{*}=l$, we have

$$
l f^{\prime}(x)=f^{\prime}(x) l^{*} \quad \text { for every } x \in \boldsymbol{E} .
$$

Now, let $a$ be a fixed element. Since

$$
l f^{\prime}(a)=f^{\prime}(a) l^{*} \quad \text { for every } l \in \mathscr{L}
$$

$f^{\prime}(a)$ cannot be in the form of $\alpha$ l. Therefore, by (9), there exists a nonzero element $b$ such that

$$
f^{\prime}(a)(b) \notin E_{b} .
$$

Since $\boldsymbol{E}_{b}$ is a closed linear subspace, there exists a non-zero element $c$ such that

$$
\left(f^{\prime}(a)(b), c\right)=0 \text { and }(b, c)=1 .
$$

Now, let $x$ be an arbitrary element and consider the linear mapping $l(y)=(y, c) x$, which is obviously symmetric. Then,

$$
\begin{aligned}
f^{\prime}(a)(x) & =f^{\prime}(a)((b, c) x)=f^{\prime}(a) l(b) \\
& =l f^{\prime}(a)(b)=\left(f^{\prime}(a)(b), c\right) x=0 .
\end{aligned}
$$

Since $x$ is arbitrary, $f^{\prime}(a)=0$, which is true for every $a \in E$. Therefore, $f=0$, because $f(0)=0$.

Now, we can prove the following theorems.

THEOREM 6. Let $f \in \mathscr{D}$. If lf is *-admissible for every $l \in \mathscr{L}$, the mapping $f$ is linear.

Proof. Let an element $a$ be fixed and let us consider the mapping

$$
g=f-f^{\prime}(a) \text {. }
$$


It is easy to see that $g^{\prime}(a)=0$ and $l g$ is *-admissible for every $l \in \mathscr{L}$. Therefore, $g$ is symmetric by (8), and it follows from Theorem 4 that

$$
(l g)^{\prime}(x)-\left((l g)^{\prime}(x)\right)^{*}=(l g)^{\prime}(a)-\left((l g)^{\prime}(a)\right)^{*}=l^{\prime}(a)-\left(l g^{\prime}(a)\right)^{*}=0,
$$

which means that $l g$ is symmetric for every $l \in \mathscr{L}$. Therefore, $g=0$ by (12), or $f=f^{\prime}(a)$; in other words, $f$ is linear.

TheoRem 7. Let $f \in \mathscr{D}$. If $f l$ is *-admissible for every $l \in \mathscr{L}$, the mapping $f$ is linear.

Proof. We consider the same $g$ as in the proof of Theorem 6. It is clear that $g^{\prime}(a)=0$ and $g l$ is $*$-admissible for every $l \in \mathscr{L}$. Therefore, for any $l \in \mathscr{L}$ such that

we have, for $a=l(b)$,

$$
a \in l(\boldsymbol{E}),
$$

$$
\begin{aligned}
(g l)^{\prime}(x)-\left((g l)^{\prime}(x)\right)^{*} & =(g l)^{\prime}(b)-\left((g l)^{\prime}(b)\right)^{*} \\
& =g^{\prime}(l(b)) l-l^{*} g^{\prime}(l(b)) \\
& =g^{\prime}(a) l-l^{*} g^{\prime}(a)=0 .
\end{aligned}
$$

In other words, we have

or

$$
g^{\prime}(l(x)) l=l^{*} g^{\prime}(l(x)) \quad \text { for every } l \in \mathscr{L} \text { such that } a \in l(E),
$$

$$
g^{\prime}(y) l=l^{*} g^{\prime}(y)
$$

for every $l \in \mathscr{L}$ such that $a \in l(E)$ and $y \in l(\boldsymbol{E})$.

Now, let $c$ be an arbitrary element and let us consider the following symmetric linear mapping

Since

$$
l(x)=x+(c, x) c .
$$

$$
l\left(y-(c, y)\left(1+\|c\|^{2}\right)^{-1} c\right)=y \quad \text { for every } y \in E,
$$

we have $l(\boldsymbol{E})=\boldsymbol{E}$. Therefore, for this mapping $l$, we have

$$
g^{\prime}(y) l(x)=\lg ^{\prime}(y)(x) \quad \text { for every } x \in \boldsymbol{E} \text { and } y \in \boldsymbol{E},
$$

which is equivalent to

or

$$
g^{\prime}(y)(x)+(c, x) g^{\prime}(y)(c)=g^{\prime}(y)(x)+\left(c, g^{\prime}(y)(x)\right) c,
$$

$$
(c, x) g^{\prime}(y)(c)=\left(c, g^{\prime}(y)(x)\right) c .
$$

Therefore, $g^{\prime}(y)$ has the following property: if $(c, x)=0$, then $\left(c, g^{\prime}(y)(x)\right)=0$. Now, let us assume that $g^{\prime}(y) \neq 0$. Then, $g^{\prime}(y)$ satisfies the condition of (9). Therefore, $g^{\prime}(y)$ should be in the form of $\alpha 1$ for some $\alpha$. However, this is impossible, because it should satisfy the equality (13). 
Remark. Let $f$ be ${ }^{*}$-admissible and $l \in \mathscr{L}$ be symmetric. Let us consider the mapping

$$
g=l f l \text {. }
$$

This is *-admissible, because, since

we have

$$
g^{\prime}(x)=l f^{\prime}(l(x)) l,
$$

which means that

$$
\begin{aligned}
\left(g^{\prime}(x)\right)^{*} & =\left(l f^{\prime}(l(x)) l\right)^{*}=l\left(f^{\prime}(l(x)) * l\right. \\
& =l\left(f^{*}\right)^{\prime}(l(x)) l=\left(l f^{*} l\right)^{\prime}(x),
\end{aligned}
$$

$$
(l f l)^{*}=l f^{*} l .
$$

Although the mappings of Hammerstein type are not always *-admissible, we can sometimes associate the mapping of the above type to the original mapping of Hammerstein type. In fact, if $l$ is a positive definite, symmetric mapping, to the mapping $l f$, which is the general form of the mapping of Hammerstein type, we can associate the mapping $l_{1} f l_{1}$ where $l_{1}$ is the square root of $l$. This method has been effectively used in $\S 10$ of [3].

\section{Ranges and null sets}

For a mapping $f$ of $\boldsymbol{E}$ into itself we denote its range and null set by $R(f)$ and $N(f)$, respectively; in other words, we put

$$
R(f)=f(E) \text { and } N(f)=\{x \in \boldsymbol{E} \mid f(x)=0\} .
$$

For a linear mapping $l$, it is well known that

$$
\begin{array}{ll}
(\overline{R(l)})^{\perp}=N\left(l^{*}\right), & \overline{R(l)}=N\left(l^{*}\right)^{\perp}, \\
\left(\overline{R\left(l^{*}\right)}\right)^{\perp}=N(l), & \overline{R\left(l^{*}\right)}=N(l)^{\perp} .
\end{array}
$$

Naturally, in the case of non-linear mappings, we cannot have such precise relations like these.

For any $f \in \mathscr{D}$, we have the following relations:

$$
R(f)^{\perp}=\bigcap_{x \in \mathbf{E}} R\left(f^{\prime}(x)\right)^{\perp}=\bigcap_{x \in \mathbf{E}} N\left(f^{\prime}(x)^{*}\right) .
$$

Proof. Let $a \in R(f)^{\perp}$. Then, for any $x \in \boldsymbol{E}$ and $y \in \boldsymbol{E}$, we have

$$
\left(a, f^{\prime}(x)(y)\right)=\lim _{\varepsilon \rightarrow 0} \frac{1}{\varepsilon}[(a, f(x+\varepsilon y)-f(x))]=0,
$$

which means that $a \in R\left(f^{\prime}(x)\right)^{\perp}$ for every $x \in E$. Conversely, if $a \in R\left(f^{\prime}(x)\right)^{\perp}$ for every $x \in \boldsymbol{E}$, 


$$
\begin{aligned}
(a, f(x)) & =\left(a, \int_{0}^{1} f^{\prime}(\xi x)(x) d \xi\right) \\
& =\int_{0}^{1}\left(a, f^{\prime}(\xi x)(x)\right) d \xi=0,
\end{aligned}
$$

hence it follows that $a \in R(f)^{\perp}$. The second equality follows immediately from (14).

For any subset $M$ of $\boldsymbol{E}$, we denote the smallest linear subset containing $M$ by $[M]$. Then, it is obvious that the following equalities follow from (15).

$$
\text { For any } \begin{aligned}
f \in \mathscr{D} \text { we have } \\
\qquad[\overline{R(f)}]=\bigcup_{x \in \boldsymbol{E}} \overline{R\left(f^{\prime}(x)\right)}=\bigcup_{x \in \boldsymbol{E}} N\left(\left(f^{\prime}(x)\right)^{*}\right)^{\perp} .
\end{aligned}
$$

On the other hand, as to the relation between $N(f)$ and $N\left(f^{\prime}(x)\right)$, we have only the following inequality.

$$
\begin{aligned}
& \text { For any } f \in \mathscr{D} \text { we have } \\
& \qquad \bigcap_{x \in \boldsymbol{E}} N\left(f^{\prime}(x)\right) \subset N(f) .
\end{aligned}
$$

Proof. If $f^{\prime}(x)(a)=0$ for every $x \in E$, since

we have

$$
f^{\prime}(\xi a)(a)=0
$$

for every $\xi$,

$$
f(a)=\int_{0}^{1} f^{\prime}(\xi a)(a) d \xi=0,
$$

which means that $a \in N(f)$.

By the relations (15), (16) and (17), the following theorem can be easily proved.

Theorem 8. Let $f$ be ${ }^{*}$-admissible. Then,

$$
\begin{array}{ll}
R(f)^{\perp} \subset N\left(f^{*}\right), & N\left(f^{*}\right)^{\perp} \subset \overline{[R(f)]}, \\
R\left(f^{*}\right)^{\perp} \subset N(f), & N(f)^{\perp} \subset \overline{\left[R\left(f^{*}\right)\right]} .
\end{array}
$$

REMARK. Each of the relations of the above theorem cannot be replaced by the equality. For example, for the mapping

$$
f(x)=\left(\xi_{1}^{2}, \xi_{1}\right) \text { where } x=\left(\xi_{1}, \xi_{2}\right)
$$

of a two-dimensional Euclidean space into itself, we have

$$
R(f)^{\perp}=\{0\} \text { and } N\left(f^{*}\right)=\left\{x=\left(\xi_{1}, \xi_{2}\right) \mid \xi_{1}^{2}+\xi_{2}=0\right\} .
$$

As is easily seen, Theorem 8 can be expressed in the following form. (18) Let $f$ be a *admissible mapping such that $R(f)$ (resp. $R\left(f^{*}\right)$ ) is closed. Then, either 
$1^{\circ}$ for any $y \in E$ there exist $x_{i} \in E(i=1,2, \cdots, n)$ and numbers $\alpha_{i}$ $(i=1,2, \cdots, n)$ such that

or

$$
\left.y=\sum_{i=1}^{n} \alpha_{i} f\left(x_{i}\right) \text { (respectively } y=\sum_{i=1}^{n} \alpha_{i} f^{*}\left(x_{i}\right)\right)
$$

$2^{\circ}$ there exists an element $a \neq 0$ such that

$$
\left.f^{*}(a)=0 \text { (respectively } f(a)=0\right) .
$$

In fact, if $[R(f)]=E$, we have $1^{\circ}$ and, if $[R(f)] \neq E$, since $R(f) \neq 0$, any non-zero element in $R(f)$ satisfies $2^{\circ}$.

\section{The mapping degree}

For the definition of the mapping degree we refer to [2], in which the following theorem has been proved:

Let $f \in \mathscr{D}$ be completely continuous (i.e., continuous and transforms every bounded set into a compact set). For a real number $\lambda$ which is not a proper value of $f^{\prime}(0)$, we consider the vector field

$$
f_{\lambda}(x)=\lambda x-f(x) \quad \text { for every } x \in E .
$$

Then, there exists a sphere $S=S_{r}=\{x \in E \mid\|x\| \leqq r\}$ such that the mapping degree $d\left(f_{\lambda}, S, 0\right)$ of $f_{\lambda}$ at 0 relative to $S$ is equal to $(-1)^{\beta}$, where $\beta$ is the sum of the multiplicities of all the proper values $\lambda^{\prime}$ of $f^{\prime}(0)$ such that $\lambda \lambda^{\prime}>0$ and $\left|\lambda^{\prime}\right|<|\lambda|$. (cf. Theorem 4.7, p. 136, [1]).

The purpose of this section is to obtain a relation between the mapping degrees of $f$ and $f^{*}$ by making use of the above theorem of Leray and Schauder. We begin with the following theorem.

THEOREM 9. Let $f$ be *admissible. Then, $f$ is completely continuous if and only if $f^{*}$ is completely continuous.

Proof. Let $f$ be completely continuous. Then, by [Theorem 7, p. 51, [3]], $f^{\prime}(x)$ is a completely continuous linear mapping for each $x \in E$. Therefore, $\left(f^{*}\right)^{\prime}(x)=\left(f^{\prime}(x)\right)^{*}$ is also completely continuous for each $x \in \boldsymbol{E}$. On the other hand, by Theorem 4 , we have

$$
f-f^{*}=f^{\prime}(x)-\left(f^{\prime}(x)\right)^{*} \quad \text { for every } x \in \boldsymbol{E} .
$$

Therefore, $f^{*}$ is completely continuous. The converse can be proved similarly.

Now, let $f$ be a ${ }^{*}$-admissible completely continuous mapping. Since $f^{*}$ is also completely continuous, $\left(f^{*}\right)^{\prime}(0)=\left(f^{\prime}(0)\right)^{*},\left(f^{\prime}(0)\right)^{*}$ has the same proper value as those of $f^{\prime}(0)$ and the multiplicities of the common proper 
values coincide, the following theorem follows from the above theorem of Leray and Schauder.

THEOREM 10. Let $f$ be $a^{*}$-admissible completely continuous mapping. For a real number $\lambda$ which is not a proper value of $f^{\prime}(0)$, let us consider the vector field $f_{\lambda}(x)=\lambda x-f(x)$ for every $x \in E$. Then,

$$
f_{\lambda}^{*}(x)=\lambda x-f^{*}(x) \quad \text { for every } x \in E,
$$

and there exists a sphere $S$ such that

$$
d\left(f_{\lambda}, S, 0\right)=d\left(f_{\lambda}^{*}, S, 0\right) .
$$

\section{References}

[1] M. A. Krasnoseliski, Topological methods in the theory of non-linear integral equations (translated by A. H. Armstrong, Pergamon Press, 1964).

[2] J. Leray and J. Schauder, 'Topologie et équations fonctionnelles', Ann. Sci. Ecole Norm. Sup. 51 (1934), 45-78.

[3] M. M. Vainberg, Variational methods for the study of non-linear operators (translated by A. Feinstein, Holden-Day, 1964).

\section{Australian National University}

Canberra 